\title{
"Age" in lung transplantation: factors related to outcomes and other considerations
}

\author{
Christopher H. Wigfield ${ }^{1}$ - Vanessa Buie ${ }^{1}$ - David Onsager ${ }^{1}$
}

Published online: 13 August 2016

(C) The Author(s) 2016. This article is published with open access at Springerlink.com

\begin{abstract}
The age of lung transplant recipients is steadily increasing. Older donors are more frequently considered. The risk factors associated with advanced age in lung transplantation warrant discussion to ensure optimal outcomes in this complex endeavor. This report provides a summary of the pertinent topics and available evidence.
\end{abstract}

Keywords Recipient $\cdot$ Donor $\cdot$ Risk $\cdot$ Outcomes $\cdot$ Lung transplantation $\cdot$ Age

"Forty is the old age of youth; fifty the youth of old age".

Victor Hugo

\section{Introduction}

Life expectancy is rising in many societies. A growing proportion of the population is over 65 years of age [1,2]. As a consequence, older patients are referred for consideration for lung transplantation [3]. Prognostic and symptomatic indications exist for this established treatment option in selected candidates with end-stage lung diseases. Lung transplantation, however, remains a complex endeavor and is offered for a

This article is part of the Topical Collection on Lungs Transplant

Christopher H. Wigfield

cwigfield@surgery.bsd.uchicago.edu

1 University of Chicago Medicine, Chicago, USA wide range of ages with associated risks (see ISHLT data graphic/Fig. 1).

This review provides summary and context for factors contributing to the discussion. Relevant definitions of "age" are offered. The limitations of currently available evidence are recognized. Analyses of registry data and other most relevant information including single center studies and retrospective cohorts are reviewed. The recipient age-related concerns are then reviewed. Additionally, we discuss the donor age-related allograft problems. Finally, a pragmatic approach to utilize such advanced aged donor lungs in appropriate recipients is considered.

\section{Background}

Age may be defined as a chronological entity or a biological concept. The numerical value ascribed to a person's age may be a vague indicator, but is frequently seen as insufficient to allow judgment regarding general health status. There is a lack of qualitative understanding in order to allow meaningful care decisions. Biological age, on the other hand, is a complex concept. It entails cell biological processes that are not easily quantified. Age related functionality of organisms has a molecular bases. Fundamentals such as a patient's tissue regenerative capacity, for example, are not currently measurable. For decisions in clinical practice, it is frequently necessary to compliment the numeric age with valid criteria for functional age.

Assessments of various factors indicative of adequacy of lung transplantation are needed in each potential recipient. Candidates over the age of 70 years are not routinely referred for workup for lung transplantation. Arbitrary restrictions exist for most programs. The conventional definition offered for "elderly" patients is older than 65 years of age (Fig. 2). United Network of Organ Sharing (UNOS) and the International 


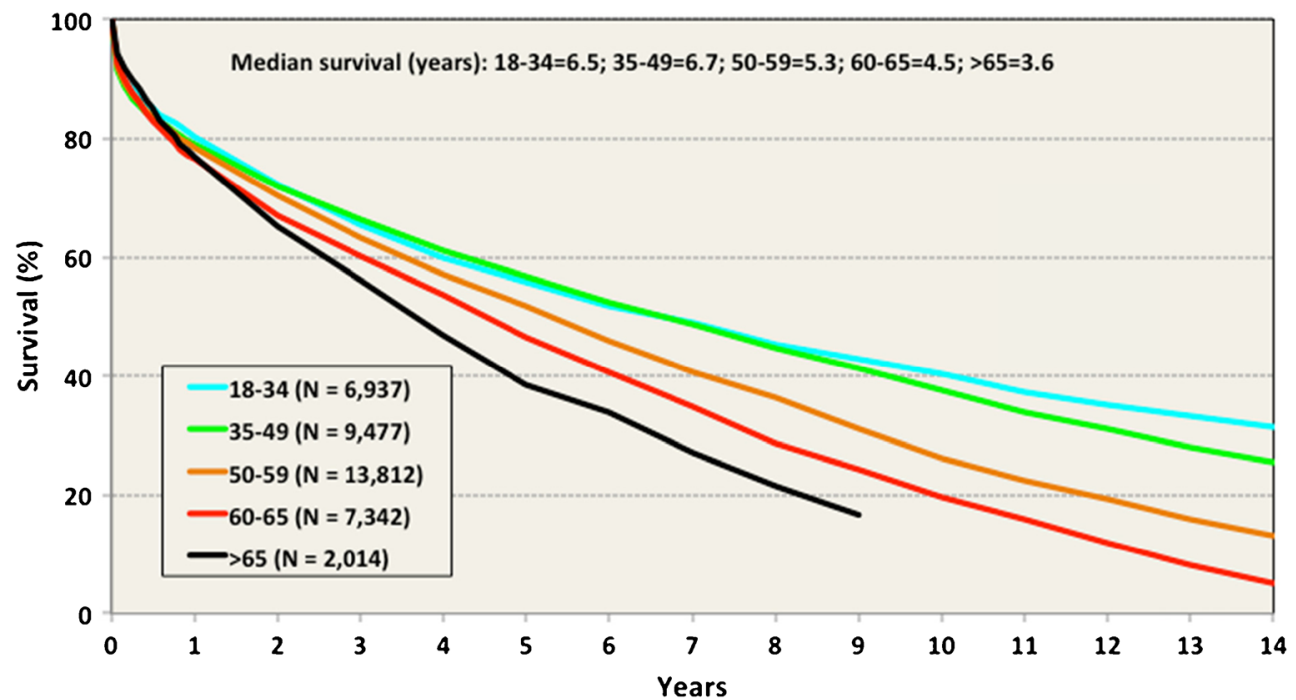

Fig. 1 Adult lung transplants. Survival by age group (transplants 1990-2011). Modified from ISHLT Registry [3]. http://www.ishlt.org/registries/slides. asp?slides=heartLungRegistry

Society of Heart and Lung Transplantation (ISHLT) offer pertinent registry data $[4,5]$. These suggest a steady shift toward older recipients over the last decade. Several centers have reported successful transplantation in patients with markedly more advanced ages.

Compared to other solid organ transplants, lung transplantation outcomes are recognized as still suboptimal [6••]. Yet, a liberation of inclusion criteria has occurred over the last decade $[7 \bullet \bullet]$. Outcomes observed, in turn, ultimately influence the allocation of allografts. This is particularly pertinent in view of the persistent shortage of adequate donor lungs. The appropriateness of lung transplantation at older ages remains a matter of debate (Fig. 1). Age, therefore, has become a focus of concern in many programs. Although not unique to lung

\section{Number of Persons 65+, 1900 to 2060 (numbers in millions)}

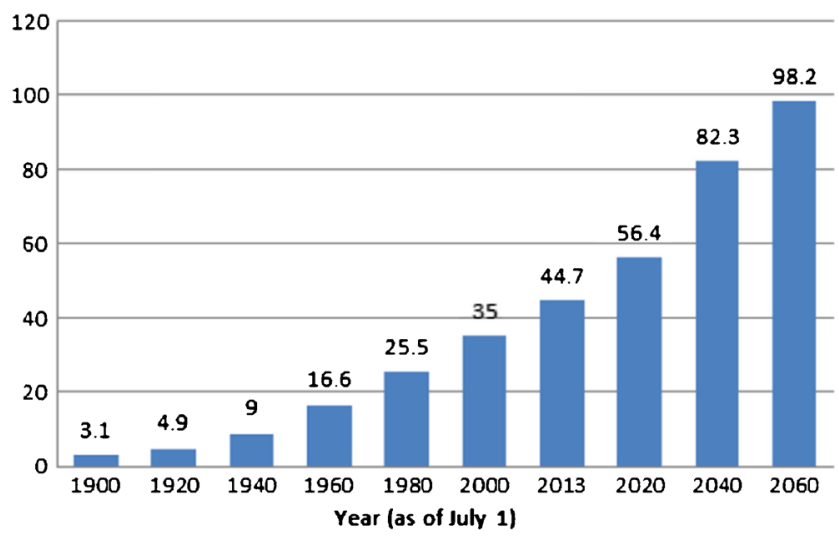

Fig. 2 Number of persons 65 years of age or older. 1900-2060 [4]. U.S Census Bureau, Population Estimates and Projections; http://www.aoa.acl. gov/Aging_Statistics/Profile/2014/2.aspx. Accessed July 6, 2015 transplantation, both recipient age-related challenges and donor age-associated factors are frequently under intense scrutiny.

\section{Phenomena related to aging}

It is generally agreed that the numeric age of some patients may not reflect their "biological" age or performance status. Determining age in biological systems, however, is a complex task. Even theoretical considerations are multifaceted. Four related phenomena have to be considered: the process of aging, the determinants of longevity, age-associated diseases encountered, and death per se [8]. The application of biological aging to the context of lung transplantation needs to acknowledge multiple clinical confounders and nonmedical lifetime limitations that pertain to individual patients.

A distinct set of genetic disorders associated with DNA repair mechanisms provided evidence regarding the need of functional genome maintenance during the aging process [9]. Bio-gerontologists have specified the determinants of aging. These are not simply driven by genetic factors. Ageing is the result of the "loss of molecular fidelity" and ensuing cellular and metabolic dysfunction [10,11]. The concept of antagonistic pleiotropy describes the beneficial role of particular genes early in life at the expense of inducing "aging" later in life [12]. Telomere changes are an area of intense research interest. Phenotypical consequences of aging, however, may not always be visible. In turn, inherited dysfunction or mutations may affect telomerases without correlation of chronological age [13].

External, i.e., environmental, triggers impact cellular regulation and immunogenic phenotype changes. The sum of these molecular processes leads to cellular and organismal senescence. Additionally, stress response research provides new 
insights into the profound alterations of normally phylogenetically preserved biochemical signaling pathways [14]. The example of the live-span shortening effect of excessive caloric intake and other specific nutritional deficits has been well described. These are cumulative in the process of ageing due to the metabolic disorders caused.

The process of ageing related general physiologic changes have been subject to considerable research efforts. Regarding lung transplant candidates, the pulmonary manifestations and systemic performance are of primary concern. Pulmonary manifestations of advancing age may aggravate any underlying respiratory disease process. The net effect may be that earlier referral for lung transplant evaluation is required. Specifically, reduced pulmonary elasticity and dependent zone airway closures are likely in the elderly. In addition, chest wall stiffness and loss of muscle mass add restrictive elements to the respiratory impairment. Physiological correlates include a reduced forced expiratory volume (FEV1) and increased residual volume, a decline in vital capacity as well as total lung capacity. Chest wall restriction and body mass index-related issues would then affect the recipient in the post-transplant phase even in the presence of a functional allograft $[15,16]$.

Other aging-related cellular adaptations have a more wideranging impact. Mitochondrial dysfunction and reduced respiratory metabolism with increased deleterious effects of reactive oxygen radicals develop. Multiple alterations of the immune system have been documented in the elderly. A decline of the adaptive immune responses and specific changes of the innate immune-system are well documented. Cellular immunity may be affected by reduced T cell subpopulations, impaired B cell generation, and non-organ-specific auto-antibody formation [17]. Susceptibility to certain pathogens has been demonstrated and hyper-reactivity of airways may develop.

Age-related specific diseases then add to the burden with individual end-organ manifestations including central nervous system and neuro-muscular and degenerative impairment. The resulting net effect of markedly reduced physiological reserve may not be apparent until a response to critical illness stressors is required. Therefore, a plethora of age-related possible comorbidities must be considered and actively investigated in elderly candidates for transplantation. Some patients will present with absolute contraindications defined for most lung transplant programs, due to excessive perioperative risks perceived and the ability to recover adequately post-operatively (Table 1 ). Other age-related disease processes are clearly identifiable with screening and manageable with adequate specialist care systems in place. As the incidence of cancer increases with age, evaluation for specific risk factors and application of screening protocols for the most common malignancies is an essential part of the assessment of elderly candidates for potential lung transplantation.

Additional concerns implicit in caring for elderly patients are the risk of failure to thrive and other systemic comorbidities difficult to reverse [18]. These include anorexia of aging and relative cognitive impairment that require detailed clinical assessment and at times specialist evaluation for underlying causes. Similar to general frailty, these entities are hard to quantify. They demand clinical acuity and judgment in the absence of reliable biomarkers.

\section{Lung transplantation in the elderly}

Life expectancy varies among societies and socioeconomic circumstances. Currently, expected remaining years of life in the US for a person reaching the age of 65 is 17.9 years for males and 20.5 years for females [19]. Lung transplantation survival rates are frequently reported for multiple age groups. National survival rate averages reported for lung transplantation in the US by UNOS are $82 \%$ at 1 year, $65 \%$ at 3 years, and $51 \%$ at 5 years, respectively. The primary motivation in lung transplants is to improve the 1-year mortality observed due to their underlying respiratory disease. Few elderly recipients, however, will have a fully restored "life expectancy". It is therefore critical to understand each individual's expectations in life and consider the symptomatic benefit for most of these patients undergoing lung transplantation.

Despite the chronological continuum, registries report outcomes observed after lung transplantation in arbitrarily selected age categories. Such analysis often considers older recipients ( $>60$ years of age and separately $>65$ years). The ISHLT registry and UNOS data provide such retrospective information regarding transplant rates and survival outcomes in these cohorts $[5,19]$. This cannot include biological variances of
Table 1 Examples for agerelated comorbidities
Possible age-related comorbidities in older lung transplant candidates

\begin{tabular}{lc}
\hline Systemic disease & $\begin{array}{c}\text { Malignancies, hypertension, cardiovascular, diabetes mellitus, } \\
\text { nutritional deficits, falls, and injuries }\end{array}$ \\
CNS disease & $\begin{array}{c}\text { Memory impairment, CVA, Parkinson's disease hearing loss, } \\
\text { vision issues, sleep disorders } \\
\text { Coronary artery disease, thyroid disease, urinary disorders, } \\
\text { arthritis, osteoporosis, GI disorders, pressure ulcers }\end{array}$ \\
\hline
\end{tabular}


patients at different ages and has to be seen as general indicators rather than meaningful for individual patient assessment.

The age of recipients, when assessed according to the era of lung transplantation in the ISHLT registry, also showed a marked increase over time for $60-65$ - and $>65$-year-old recipients $[20 \bullet \cdot$. This trend of increasing median age for lung transplant candidates has not been affected by implementation of the Lung Allocation Score (LAS) in the US [21]. The proportion of the 60-69-year-old recipients in particular has steadily increased over the last 15 years according to UNOS 2014 data.

Single-center reviews provided limited evidence for survival outcomes in "older" patient cohorts. These studies used variable age cutoffs and are not directly comparable with meta-analysis [22]. Notable in some of these cohorts are the higher incidence of lethal infective complications in such older recipients. Reviewing the complication rates of elderly recipients, Tomascek et al. reported specific patient characteristics associated with impaired outcomes in a single center review. These included postoperative complications, length of stay, achieved FEV1, and development of BOS at 1 year. BMI was identified to impact overall complication rates in their cohort older than 60 years at time of transplantation [23]. Body composition and frailty have not been considered in these reviews. No useful biomarkers or surrogate parameters were available at the time of these retrospective studies.

A report reviewing available SRTR data indicates that 1year survival for septuagenarians in lung transplantation is significantly worse when compared with 60-69-year-old recipients in the cohort prior to 2005 ; (57 vs. $76 \% 1$-year survival, $\log \operatorname{rank} p=0.01$ ) [24]. Three- and 5-year survival was noted to be significantly less for elderly lung transplant recipients. There appears to have been an improvement of survival in septuagenarians since the LAS inception in the USA (Fig. 3). This may be manifest despite increased proportion of idiopathic pulmonary fibrosis in this cohort. The cohort comprised 225 recipients with an observed one-year survival of $79 \%$. Due to their relative improvement, advanced age was not considered to be an "absolute" contraindication [24].

A comparison of older (defined as $>60$ years of age) to younger recipients in the Toronto Lung Transplant program published in 2007 showed a marked 1-year survival difference (60 vs. $86 \%$ ). A difference in survival outcome was also noted at 5 years ( 37 vs. $57 \% ; p=0.005)$. The small number of complete 5 years follow-up for recipients was among the limiting factors in this and other similar single center studies published to date. Infectious complications were the predominant cause for early deaths noted in this study. It also noted the excess mortality for elderly recipients despite adjustment for the Declining Exponential Approximation to Life Expectancy (DEALE) in the older recipient group [23].

Vadnerker et al reviewed age-related complications in recipient older than 65 years in 2011. A higher incidence of malignancy and acute cellular rejection requiring augmented immunosuppression was detected in this particular cohort compared with the institutional experience of 60-65-yearold recipients [25]. The associated editorial by Rahman et Cahill points out that there was no detectable short-term survival difference in this recent cohort with an overall relatively high rate of complications. The lack of disease-specific analysis and long-term survival of elderly recipients is apparent.

Case reports provide limited insight into current practice of individual lung transplant programs. Shigemura et al. reported lung transplantation for a single octogenarian with good early outcome but concede that "A number of complicated issues remain debatable, including ethical concerns..." Clearly, the appropriate allocation of suitable allografts as a scarce commodity remains a concern.

A more recent analysis of the ISHLT registry data compared the median survival of recipients separated into five age groups. The Kaplan-Meier survival curves provided to show a marked separation after 12 months for recipients older than 60 years old. There is a further attrition rate apparent when comparing the subgroup older than 65 years old ( $n=$ 2014 ) with all other recipient age groups (age groups in years $<34, n=6937$; 35-49, $n=9477 ; 50-59, n=13812 ; 60-65$, $n=7342$ ). This represents the best registry evidence available to guide transplant programs. The retrospective nature of the analysis limits the value for individual case management, as it does not adjust for other selection criteria. The precise impact of age on prognostic value of lung transplantation would require prospective studies with surrogate markers for agerelated conditions in this context.

\section{Donor age impact in lung transplantation}

The dichotomy of the transplant process is evident when considering the donor-related circumstances. The prevalence of pulmonary diseases among the ten leading causes of death must be recognized. Ruling out pulmonary malignancy, chronic obstructive airway disease and pneumonia may not be simple in the donor setting. Furthermore, viral respiratory tract infections have seasonal variation with higher prevalence in senior donors with little clinical correlation available.

It is noteworthy that currently no regulatory restrictions are imposed on acceptance of older donor lungs. Individual lung transplant programs generally follow selection criteria protocols. Current lung allocation systems do not incentivize procurement of older donor lungs and utilization rates remain low. Nevertheless, the impact of numerous donor parameters has been studied to assess the possible impact on outcomes after lung transplantation [26, 27]. The donor age variable features in several retrospective analyses.

Satisfactory outcomes can be achieved with older lung allografts, but the impact of age on physiologic changes of lung function must be appreciated [15]. Decreased elasticity 
Age trends in lung transplantation over the last decade

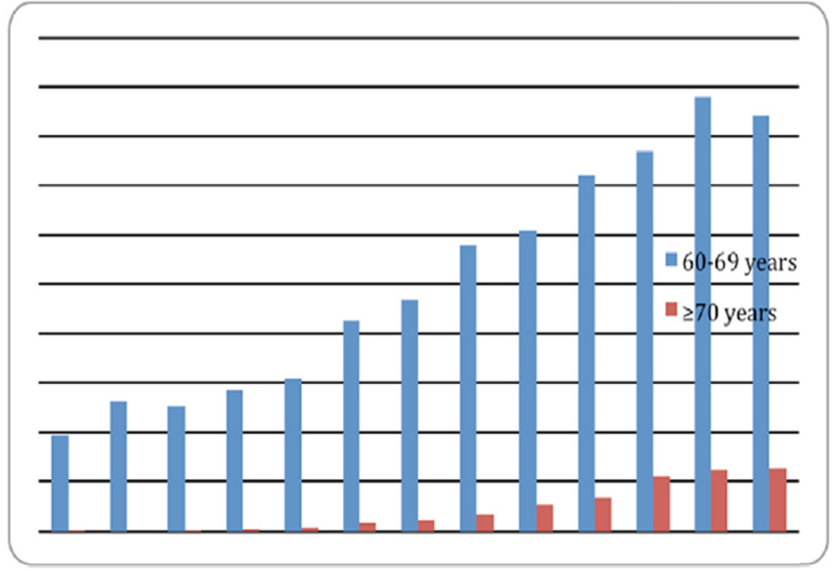

Fig. 3 Trends in lung transplantation over the period of 2000 to 2012 for septuagenarians and sexagenarians in the USA, modified from Hayanga et al. [24]

with resulting residual volume increase and vital capacity decrease may be a concern. Such parameters and likely increased A-a $\mathrm{O}_{2}$ gradients of older lungs may not be quantifiable in the procurement situation. Potential chest wall stiffness and muscle mass reduction of older donors, however, may not have a clinically important impact on total lung capacity and maximum inspiratory pressures, as they arguably depend more on the recipient's condition in the peritransplant period.

In biological terms, the immunology of advanced age donor lungs warrants some attention. The decline of adaptive immune system function and the changed state of the innate immune responses will affect the lung allograft. These changes are not quantifiable in current routine procurement practice but may render older allografts to higher susceptibility to particular respiratory tract infections and therefore increase risks as inherited by the recipient [17]. Donors of advanced age have been shown to express lower levels of interleukin-10 early after reperfusion. Cytokine gradients in turn are known to correlate with the development of primary graft dysfunction. The risk of primary graft dysfunction in the absence of rejection and cardiogenic issues or infective pathogens causing graft impairment has been studied.

Although early outcomes of utilization of older lungs have been shown to be similar to those in recipients of lungs from younger donors, late survival and the development of bronchiolitis obliterans syndrome have been negatively impacted. There is currently no correlation of different donor age groups to the various chronic lung allograft dysfunction (CLAD) phenotypes observed.

The influence of donor and recipient age in lung transplantation was studied by Hayes et al. with a review of the United Network of Organ Sharing (UNOS) SRTR registry data. There is evidence that the number of older donor's allografts being utilized for transplantation is increasing [5]. Multivariate Cox models applied to the large cohort demonstrated that donors below 50 years of age or over reduced the recipients mortality if less than 60 years old at time of transplantation. If, however, the recipient was over the age of 65 , only donors less than 50 years old decreased the risk for death after transplantation. The primary finding was that older donor lungs appeared not to affect survival when transplanted for older recipients but limited outcomes in "younger recipients." Such data suggests that in order to optimally utilize the donor pool, older donor lungs should be used but some donor-recipient age matching should be considered. This approach may potentially enhance longterm outcomes in specific recipient age subgroups. It presents an additional challenge in the complex matching of allografts. After exclusion of significant functional impairment, and in addition to serology, immunology, and size, age similarity in older could be advocated in such recipients.

"Age-range matching" allocation of older lung allografts ( $>60$ years old) may provide a pragmatic approach to increase the utilization rate of these organs offered but frequently rejected. A recently published "focus theme age" in lung transplantation analyzed the information available in the ISHLT registry. Two-thirds of recipients are aged 45-65 years, but the trend for 60-69 years old and older than 70 years old is increasing (see Fig. 3). Extremes of age in recipients confer an independent risk factor for early death after transplants. The model utilized in this retrospective analysis also demonstrates older donor age to be an independent risk factor associated with mortality after lung transplantation $[20 \bullet \cdot]$.

\section{Conclusions}

Age will increasingly factor in the decision-making process for lung transplant candidates. When considering patients for the rigors of lung transplantation, life expectancy must not be confused with individuals' expectations in life. Frequently, patients are seeking symptom relief and the known prognostic limitations germane to lung transplantation may appear acceptable to older candidates. Informed consent obtained from such candidates ought to include a specific benefit versus risk discussion. For established subgroups of patients with endstage respiratory disease who have a reasonable survival advantage with transplantation, the systemic aspects inherent with aging cannot be ignored.

The reported 1-year outcomes available from SRTR and ISHLT registries are currently the best source of information for generic decisions. Future analyses ought to further study the impact of age on outcomes in lung transplantation. The overriding concern, to allocate scarce lung allografts to the most appropriate recipients, has to include the assessment of the patient's potential for full recovery, irrespective of "chronological" age at the time of transplantation. Threeyear survival outcomes observed in the subpopulation 
transplanted at age 65 or older remain inferior to those observed for younger age categories. Lungs from donors with a chronological age of 45 and older can safely be transplanted, but the stakes may be higher for such allografts especially in combination with other extended donor criteria.

The advent of ex vivo lung perfusion systems (EVLP) may allow for detailed differential assessment of older donor lungs. This may lead to separation and selective use of "good old lungs." Interventions aimed at regenerative or repair mechanisms may be feasible to improve lung procurement to optimize aged allografts.

A key concern remains who may be the most appropriate recipient for any given organ to enhance outcomes. The matching of older donors with advanced age recipients may be a reasonable way of increasing utilization rates of such allografts available. This may help to a modest extent to alleviate the lung allograft shortage and serve the increasingly listed and carefully selected elderly candidates for lung transplantation.

In light of the current evidence available, we advocate for age matching of older donor lungs to optimize utilization with a pragmatic approach.

\section{Compliance with ethical standards}

Conflict of interest David Onsager and Vanessa Buie declare no conflicts of interest.

Christopher Wigfield declares a stipend as the educational affairs director at the International Society for Heart and Lung Transplantation.

Human and animal rights and informed consent This article does not contain any studies with human or animal subjects performed by any of the authors.

Open Access This article is distributed under the terms of the Creative Commons Attribution 4.0 International License (http:// creativecommons.org/licenses/by/4.0/), which permits unrestricted use, distribution, and reproduction in any medium, provided you give appropriate credit to the original author(s) and the source, provide a link to the Creative Commons license, and indicate if changes were made.

\section{References}

Papers of particular interest, published recently, have been highlighted as:

- O f importance

-Of major importance

1. United Nations, Department of Economic and Social Affairs, Population Division. World Population Ageing 2013. ST/ESA/SER.A/348

2. Grayson VK, Velkoff VA, et al. The Next Four Decades. The Older Population in the United States: 2010 to 2050. May 2010. http://www. aoa.acl.gov/Aging_Statistics/future_growth/DOCS/p25-1138.pdf. Accessed April 2015.
3. Lund $\mathrm{L}$ et al. The Registry of the International Society for Heart and Lung Transplantation: Thirty-second Official Adult Lung and Heart Lung Transplantation Report; Focus Theme: Early Graft Failure The International Society for Heart and Lung Transplantation. J Heart Lung Transplant. 2015;34(10)1264-1277.

4. Administration on Aging, U.S. Department of Health and Human Services. A Profile of Older Americans: 2009. http://www.aoa. gov/AoARoot/Aging_Statistics/Profile/2009/4.aspx. Accessed 31 December 2016.

5. Valapour et al. OPTN/SRTR 2014 Annual Data Report. Am J Transplant. 2016; 16(S2):141-168. http://srtr.transplant.hrsa. gov/annual_reports/ Accessed Oct 20152015.

6. Opelz $\mathrm{G}$ et al. The collaborative transplant study registry. Transplant Rev. 2013;27:43-5. The Collaborative Transplant Study (CTS) followed outcomes of solid organ transplants over the last 30 years. It serves comparative evaluation of over half a million kidney, liver, heart, lung, and pancreas transplant procedures. This registry serves as international reference source in the field of solid organ transplantation.

7.• Weill D et al. A consensus document for the selection of lung transplant candidates: 2014 - An update from the Pulmonary Transplantation Council of the International Society for Heart and Lung Transplantation. J Heart Lung Transplant. 2014;34(1):1-15. Update from the ISHLT consensus group to support decision making process in candidate selection for lung transplantation. Guidelines are provided with best evidence available and expert opinions include statements regarding standard criteria in lung transplantation. Emphasis on need for systematic evaluation and outcome observations in registries with consideration for numerous criteria, including age.

8. Bowen RL, Atwood CS. Living and dying for sex. A theory of ageing based on the modulation of cell cycle signaling by reproductive hormones. Gerontology. 2004;50(5):265-90.

9. Cicca A, Ellege SJ. The DNA damage response: making it safe to play with Knives. Mol Cell. 2010;40(2):179-204.

10. Hayflick L. Biological ageing is no longer an unsolvable problem. Ann N Y Acad Sci. 2007;1100:1-13.

11. Kirkwood TB. Understanding the Odd Science of Aging. Cell. 2005;120(4):437-47.

12. Blagosklonny MV. Revisiting the antagonistic pleiotropy theory of aging: TOR-driven program and quasi-program. Cell Cycle. 2010;9(16):171-3176.

13. Calado TR, Young NS. Mechanisms of disease: telomere diseases. N Engl J Med. 2009;361:2353-65.

14. Halgis MC, Yankner BA, et al. The aging stress response. Mol Cell. 2010. doi:10.1016/j.molcel.2010.10.002.

15. Criner JC, D’Alonzo GE. Pulmonary Pathophysiology. Madison: Fence Creek Publishing; 1999.

16. Lal-Fook SJ, Hyatt RH. Effects of age on elastic moduli on human lungs. J Appl Physiol. 2009;89:163-8.

17. Meyer KC. The role of immunity in susceptibility to respiratory infection in the aging lung. Respir. 2001;128:23-31.

18. Sarkisian C, Lachs M. "Failure to thrive" in older adults. Ann Intern Med. 1996;124(12):1072-8.

19. National Vital Statistics System, Mortality. National Center for Health Statistics. 2012. http:/www.cdc.gov/nchs/data_access/vitalstatsonline. htm. Accessed 9 Oct 2015

20.• Yusen RD et al. The Registry of the International Society for Heart and Lung Transplantation: Thirtieth Adult Lung and Heart-Lung Transplant Report - 2013; Focus Theme: Age. J Heart Lung Transplant. 2013;32(10):965-78. ISHLT Registry report presents data from over $\mathbf{4 0 0 0 0}$ adult lung transplants. This report describes donor and recipient characteristics, transplant type and recipient outcomes data. The Registry slide set is available 
online (www.ishlt.org/registries). This report focuses on recipient and donor age related data.

21. Kilic A, Merlo CA, Conte JV, Shah AS. Lung transplantation in patients 70 years old or older: have outcomes changed after implementation of the lung allocation score? J Thorac Cardiovasc Surg. 2012. doi:10.1016/j.jtcvs.2012.07.080.

22. Gutierrez $\mathrm{C}$ et al. The effect of recipient's age on lung transplant outcome. Am J Transplant. 2007;7:1271-7.

23. Tomaszek SC et al. Outcome of lung transplantation in elderly recipients. Eur J Cardiothorac Surg. 2011;39(5):726-31.
24. Hayanga AW, Aboagye JK. Contemporary analysis of early outcomes after lung transplantation in the elderly using a national registry. J Heart Lung Transplant. 2015;34:182-8.

25. Vadnerkar A et al. Age-specific complications among lung transplant recipients 60 years and older. J Heart Lung Transplant. 2011;30(3):273-81.

26. Rosengard BR, Garrity ER, Love RB, et al. Report of the Crystal City. Am J Transplant. 2002;2:701-11.

27. Orens JB et al. A review of lung transplant donor acceptability criteria. J Heart Lung Transplant. 2003;33(11):1183-200. 